\title{
Critérios de qualidade de banana Cavendish para comercialização: Rede varejista da
}

\section{Região Alto Tietê}

\author{
Cavendish banana quality criteria for commercialization: Retaill chain of Alto Tietê Region
}

Criterios de calidad para la comercialización del banano Cavendish: Cadena minorista de la Región del Alto Tietê

Recebido: 13/12/2021 | Revisado: 21/12/2021 | Aceito: 25/12/2021 | Publicado: 05/01/2022

Grasiéle Émili Ferreira de Morais

ORCID: https://orcid.org/0000-0003-0813-8939 Instituto Federal de Educação, Ciência e Tecnologia de São Paulo, Brasil

E-mail: grasiele.emili@gmail.com

Adriano Maniçoba da Silva

ORCID: https://orcid.org/ 0000-0002-0094-404X Instituto Federal de Educação, Ciência e Tecnologia de São Paulo, Brasil E-mail: adrianoms@ifsp.edu.br

Régis Cortez Bueno

ORCID: https://orcid.org/0000-0002-2923-4930 Instituto Federal de Educação, Ciência e Tecnologia de São Paulo, Brasil E-mail: regiscb@ifsp.edu.br Wilson Yoshio Tanaka

ORCID: https://orcid.org/ 0000-0002-1084-6566 Instituto Federal de Educação, Ciência e Tecnologia de São Paulo, Brasil E-mail: w.tanaka@ifsp.edu.br

Eugenio de Felice Zampini

ORCID: https://orcid.org/0000-0001-6569-8460 Instituto Federal de Educação, Ciência e Tecnologia de São Paulo, Brasil E-mail: eugenio.zampini@ifsp.edu.br

João Gilberto Mendes dos Reis

ORCID: https://orcid.org/0000-0001-6409-2299 Universidade Paulista, Brasil

E-mail: joao.reis@docente.unip.br

Sivanilza Teixeira Machado

ORCID: https://orcid.org/0000-0003-2746-7885

Instituto Federal de Educação, Ciência e Tecnologia de São Paulo, Brasil

E-mail: sivateixeira@yahoo.com.br

\section{Resumo}

A classificação de qualidade de frutas e vegetais é utilizada para selecionar produtos seguros para o consumo humano. Assim, este trabalho tem como objetivo investigar o sistema de classificação da qualidade da cadeia produtiva da banana no varejo e no atacado da região do Alto Tietê, Brasil. Foi coletado 239 bananas dos 12 principais varejoatacadistas e aplicado os Critérios de Classificação da Banana, do Programa Brasileiro de Modernização da Horticultura e Produção Integrada de Frutas. Portanto, foram considerados os critérios de qualidade: peso bruto e líquido, comprimento, calibre, grau de maturação e número de defeitos. Observou-se que a banana apresentou uma boa classificação com média de peso bruto e líquido respectivamente 156,49 $\pm 39,62$ e 98,93 $\pm 30,11$, comprimento $21,11 \pm 2,35$, calibre 37,06 $\pm 3,08$. Ainda, notou-se associação entre os critérios de qualidade e a classificação das bananas (teste de Dunn, $p<0,05)$, bem como, entre os tipos de defeitos e lotes analisados $\left(\chi^{2}=98,11 ; p<0,05\right)$. Do total de bananas analisadas, $38 \%$ estavam isentas de defeitos, mas $62 \%$ apresentaram algum tipo de defeito. Além disso, foi proposto uma estrutura estratégica para a destinação correta de bananas, como: 44\% das bananas devem ser enviadas para a produção de compostagem, 17\% para a indústria de ração animal, 1\% para a produção de alimentos processados e $38 \%$ para a comercialização no mercado para consumo humano. Concluiu-se que o processo de classificação auxilia na transparência da cadeia produtiva de alimento, e que o monitoramento e controle da qualidade devem ser contínuos durante a exposição dos frutos para venda ao consumidor, garantindo a segurança alimentar, redução do desperdício e destinação correta das bananas não aptas para comercialização.

Palavras-chave: Bananicultura; Cadeia produtiva de alimentos; Controle da qualidade; Monitoramento do varejoatacadista. 


\begin{abstract}
The quality classification of fruits and vegetables is used to select safety products for human consumption. Thus, this paper aims to investigate the quality classification system of the banana supply chain offered in retail and wholesale in Alto Tietê Region, Brazil. We collected 239 bananas from 12 main retail-wholesaler and applied the Banana Classification Criteria, of the Brazilian Modernization of Horticulture Program \& Integrated Fruit Production. Therefore, we considered the quality criteria: gross and net weight, length, caliber, degree of maturation, and the number of defects. We observed that the banana showed a good classification with an average of gross and net weight respectively $156.49 \pm 39.62$ and $98.93 \pm 30.11$, length $21.11 \pm 2.35$, caliber $37.06 \pm 3.08$. We found an association among quality criteria and bananas classification (Dunn test, $p<0.05$ ), as well as, among kind of defects and lots analyzed $\left(\chi^{2}=98.11 ; p<0.05\right)$. Thirty-eight percent of the total bananas evaluated were free of defects, but $62 \%$ showed some kind of defects. In addition, considering the banana classification process we proposed a framework correct destination, such as $44 \%$ of banana should be sent to the organic fertilizer industry, $17 \%$ to the animal feed industry, $1 \%$ to the human food industry, and $38 \%$ to fresh sales in the market to human consumption. We concluded that the classification process help in the transparency of the food supply chain and the quality monitoring and control should be continuous during the fruits are exposed to sale to the consumer, guarantee the food safety, waste reduction, and correct destination of bananas that are not good for commercialization.
\end{abstract}

Keywords: Banana farming; Food supply chain; Quality control; Retail-Wholesale Monitoring.

\title{
Resumen
}

La clasificación de calidad de frutas y verduras se utiliza para seleccionar productos de seguridad para el consumo humano. Así, este estudio tiene como objetivo investigar el sistema de clasificación de la calidad de la oferta de la cadena de suministro de banano en minoristas y mayoristas en la región de Alto Tietê, Brasil. Recolectamos 239 bananos de 12 principales minoristas y mayoristas y se aplicó los Criterios de Clasificación de Bananos del Programa Brasileño de Modernización de la Horticultura y Producción Integrada de Frutas. Por tanto, se consideraron los criterios de calidad: peso bruto y neto, longitud, calibre, grado de maduración y número de defectos. Se observó que el banano presentó una buena clasificación con un promedio de peso bruto y neto respectivamente 156,49 $\pm 39,62$ y $98,93 \pm 30,11$, longitud $21,11 \pm 2,35$, calibre 37,06 $\pm 3,08$. Se encontró asociación entre criterios de calidad y clasificación de banano (Dunn, $p<0,05$ ), así como entre tipos de defectos y lotes analizados $\left(\chi^{2}=98,11 ; p<0,05\right)$. El $38 \%$ del total de bananos evaluados estaba libre de defectos, pero el $62 \%$ presentaba algún tipo de defecto. Además, considerando el proceso de clasificación del banano, se propuso un marco para corregir el destino, como por ejemplo: $44 \%$ del banano debe enviarse a la industria de fertilizantes orgánicos, $17 \%$ a la industria de alimentos para animales, $1 \%$ a la industria de alimentos humanos y $38 \%$ a productos frescos ventas en mercado para consumo humano. Se concluyó que el proceso de clasificación ayuda en la transparencia de la cadena de suministro de alimentos y el seguimiento y control de la calidad debe ser continuo durante la exposición de las frutas a la venta al consumidor, garantizar la seguridad alimentaria, la reducción del desperdicio y el destino correcto de los bananos no buenos para la comercialización.

Palabras clave: Cultivo de banano; Cadena de producción de los alimentos; Control de calidad; Monitoreo minoristamayorista.

\section{Introdução}

A padronização de alimentos é um processo desafiador para a indústria de transformação, mesmo em ambiente controlado e o processamento atendendo critérios de qualidades pré-estabelecidos. Entretanto, mais desafiador se torna para a produção de alimentos in natura produzidos em campos abertos e submetidos as variáveis climáticas e condições da terra. O conceito de qualidade aplicado no contexto de cadeias produtivas de alimentos globais não é recente. Entretanto, os conceitos de qualidade para cadeias hortifrúti ainda tem sido pouco discutidos, principalmente, em países em desenvolvimento como o Brasil.

A busca pela padronização do processo de produção de produtos agropecuários e por maior produtividade, com aplicação de agricultura de precisão tem sido constante (Franco et al., 2017; Lindblom et al., 2017), principalmente por não se ter o controle dos diversos fatores que impactam no ambiente de produção desses produtos, que apresentam alta variação no padrão de qualidade. Neste contexto, é importante a compreensão das particularidades de cada cultura, como exemplo a cadeia produtiva da bananicultura.

A produção de banana é destaque na agricultura brasileira. Em 2020, o país produziu 6,7 milhões de toneladas com rendimento de 14,72 ton/ha (Instituto Brasileiro de Geografia e Estatística - [IBGE], 2020). De acordo com a Confederação da 
Research, Society and Development, v. 11, n. 1, e22311124679, 2022

(CC BY 4.0) | ISSN 2525-3409 | DOI: http://dx.doi.org/10.33448/rsd-v11i1.24679

Agricultura e Pecuária do Brasil - CNA (2020), em 2020, o agronegócio teve participação de 26,6\% no Produto Interno Bruto brasileiro e entre as culturas com crescimento no ano, a banana se destacou com 3,62\%. Soares et al. (2020), relatam que a banana é um dos alimentos mais consumidos e apresenta valor nutricional e teor energético elevado. Sobre a comercialização da banana, Soares et al. (2020), relatam que por tratar-se de uma fruta climatérica, o manejo pós-colheita deve ser rápido e cuidadoso para não perder a qualidade do fruto.

O monitoramento da qualidade de produtos alimentícios é crucial devido os seus efeitos na saúde humana. A hortifruticultura brasileira tem evoluído nos últimos anos e vem demonstrando desempenho cada vez melhor. Todavia, ainda precisa de muitos avanços para reduzir os altos índices de perdas e desperdícios. E contribuindo para os avanços dos estudos de padrões de qualidade do fruto tem-se como referência as Normas de Classificação da Banana, do Programa Brasileiro para Modernização da Horticultura \& Produção Integrada de Frutas - [PBMH \& PIF], proposto pela Companhia de Entrepostos e Armazéns Gerais de São Paulo - [CEAGESP] (PBMH \& PIF, 2006). A Ceagesp realiza estudos sobre a classificação dos produtos hortícolas, com o objetivo de apresentar regras claras e permitir a transparência no processo de comercialização nos entrepostos, assim, são 43 normas de classificação de frutas e hortaliças além de 97 padrões mínimos de qualidade e medidas de frutas e hortaliças (CEAGESP, 2021).

O objetivo da classificação é o controle de qualidade de produtos para garantir a oferta de alimentos saudáveis ao consumidor e, quando realizada de maneira correta, deve além de auxiliar na comercialização e possibilitar a seleção de produtos para diferentes usos, em função da qualidade, com diferenciação de preços e redução de despesas na comercialização (CEAGESP, 2021). O conceito de qualidade aplicado na cadeia de alimentos permeia sobre os aspectos intrínsecos e extrínsecos que envolvem a produção, transformação e distribuição dos produtos alimentícios (Trienekens \& Wognum, 2013; Hsiao et al., 2017; Yap et al., 2017). Nos estudos de Mannes et al. (2018) fica evidenciado a importância da aplicação das ferramentas da qualidade no setor alimentício para atender questões sanitárias e de mercado.

A qualidade dos produtos hortifrúti, seja in natura ou beneficiados, depende do desenvolvimento e aplicação de métodos que atendam as características da cadeia, garantindo a segurança alimentar e valor agregado à cadeia. De acordo com Macheka et al. (2017), os principais desafios da logística aplicada as cadeias hortifrúti estão no controle das atividades de planejamento da quantidade de colheita, de processamento, do nível de estoque, da seleção do modal de transporte, da programação de transporte, enquanto que o controle das atividades da qualidade estão na determinação do grau de maturação do hortifrúti e do momento da colheita, práticas aplicadas na colheita, na decisão sobre os padrões de classificação, as práticas de classificação, seleção do material de embalagem, práticas de embalagem, monitoramento das condições de estocagem e as práticas de estocagem, monitoramento das condições do transporte, etc.

Neste contexto, o sistema de classificação contribuirá com a tomada de decisão não só do nível de qualidade do produto, mas para a correta destinação do produto otimizando as operações logísticas, reduzindo tempo e retrabalho, taxas de devoluções de produtos por padrões de qualidade inadequados e redução das taxas de perdas e desperdícios gerados pela cadeia. A classificação das frutas é uma atividade que representa a conexão entre os elos da cadeia, pois a aceitação do padrão das frutas é que permitirá o fluxo entre a cadeia, seguindo o produto da ponta de fornecimento até o mercado consumidor.

$\mathrm{O}$ abastecimento de alimentos frescos dos grandes centros urbanos, cita-se como exemplo, São Paulo e Rio de Janeiro é um desafio constante para a cadeia complexa, devido ao envolvimento de produtores, freteiros, atravessadores, centrais atacadistas, distribuidores e as diversas modalidades do serviço de alimentação e de varejo (Almeida, 2021). Rocha et al. (2018), apontam que a taxa de deterioração da qualidade nutricional está relacionada à modificação do sabor, mudança da textura e da aparência, muitas vezes ocasionadas pelos danos fisiológicos devido as operações desde a colheita até a distribuição e associada as condições climáticas adversas.

O perfil do consumidor atual vem alterando as formas de negociação e comercialização de produtos, pressionando as 
cadeias por práticas cada vez mais sustentáveis, produtos com melhor qualidade, segurança e informação sobre os produtos, preço acessíveis etc. (Kondo, 2010; Trienekens \& Wognum, 2013). Dessa forma, a classificação do produto é uma atividade chave que deve ser estudada e aprofundada, pois permite uma remuneração justa e adequada ao produtor, sendo este pago pela qualidade do seu produto (Kondo, 2010). Por isso, é uma atividade estratégica dentro da cadeia produtiva de bananicultura.

Dessa forma, este artigo teve como objetivo analisar a situação atual do sistema de classificação da cadeia produtiva de bananicultura na Região do Alto Tietê, tendo como propósito a destinação correta dos alimentos.

\section{Metodologia}

\subsection{Caracterização da pesquisa e procedimento metodológico}

A região do Alto Tietê é responsável pelo abastecimento de diversas regiões, conhecida como cinturão verde de São Paulo, pela forte atuação da cadeia produtiva de hortifruti. A região é composta por doze municípios, a distribuição da população estimada superior 3,0 milhões de habitantes (IBGE, 2018).

A cadeia produtiva de hortifruti é composta por produtores de frutas, legumes, tubérculos e verduras. Dessa forma, a banana foi selecionada para aplicação do procedimento metodológico, visto representar uma das frutas mais consumidas pelos brasileiros e por ser produto considerado de entrada direta nos pontos de varejos (supermercados) da região (PBMH \& PIF, 2006; Soares et al., 2020). Com o objetivo de analisar a banana comercializada na região do Alto Tietê, por meio de critérios de controle de qualidade, foi realizada uma pesquisa de campo com abordagem mista. Perovano (2016), destacou que as pesquisas mistas têm como foco a qualitativa e quantitativa ou apenas um deles, mas que seus aspectos podem ser analisados concomitantemente.

Para realizar a pesquisa em campo foi necessário selecionar o produto e estabelecer os critérios de qualidades, sendo utilizado um checklist elaborado com base nas Normas de Classificação da Banana, do Programa Brasileiro para Modernização da Horticultura \& Produção Integrada de Frutas (PBMH \& PIF, 2006) e revisão de literatura sobre as características do processo de classificação (requisitos de qualidades) dos produtos da cadeia de hortifruti (Camargo Filho \& Camargo, 2019). $\mathrm{O}$ instrumento de coleta de dados utilizado na pesquisa deve estar diretamente vinculado às variáveis previstas, com a finalidade de gerar análise e discussão sobre o observado (Perovano, 2016). Neste contexto, o checklist utilizado foi composto por quatro partes distintas: 1) identificação do documento e dados sobre a unidade de coleta de dados; 2) características e referências de qualidade para avaliar e classificar a banana Cavendish;3) Amostragem; e 4) características observadas.

A coleta de dados foi realizada em 11 de agosto de 2021, em 12 estabelecimentos abrangendo os seguintes municípios: Biritiba Mirim, Guarulhos, Itaquaquecetuba, Mogi das Cruzes, Poá e Suzano. Neste estudo, adotou-se como lote homogêneo a quantidade de bananas expostas ao cliente no varejista na data de coleta, podendo ser formada por uma banca e/ou gondola. Para composição da amostra foram coletados 20 dedos de bananas por lote homogêneo de cada estabelecimento, considerando a disposição apresentada no Quadro1.

Quadro 1: Plano de coleta de amostra por lote homogêneo.

\begin{tabular}{|c|c|c|}
\hline $\mathrm{L}_{\mathrm{i}}(4)$ & \multirow{2}{*}{$\mathrm{L}_{\mathrm{i}}(4)$} & $\mathrm{L}_{\mathrm{i}}(4)$ \\
\cline { 1 - 1 } $\mathrm{L}_{\mathrm{i}}(4)$ & & $\mathrm{L}_{\mathrm{i}}(4)$ \\
& & \\
\hline
\end{tabular}

Fonte: Autores (2021).

Onde, $\mathrm{Li}=$ local de coleta, com i variando de 1 a $5 ;(4)$ = quantidade de dedos retirados de cada $\mathrm{Li}$. 
Os critérios de qualidade avaliados foram relacionados as caraterísticas externas, tais como classe (comprimento externo do dedo, cm), subclasse (escala de maturação), calibre mínimo, peso bruto (fruto e casca), peso líquido (fruto), peso da casca, defeitos observados (categorias Extra, I, II e III).

Para avaliação dos dedos de banana foi utilizado uma balança e fita métrica. Com a balança conduziu-se a pesagem dos dedos de banana, obtendo o peso bruto (Pr) e peso da casca (Pc), em gramas. O peso líquido (Pl) foi calculado a partir da diferença entre o peso bruto e o peso da casca. Da mesma forma, a fita métrica foi aplicada para a medição do comprimento externo $($ em $\mathrm{cm})$ e da circunferência da banana (em mm). Para a medição dos dedos e verificação das classes, utilizou-se o comprimento externo do dedo de banana, já para a circunferência mediu-se a parte central da banana, Figura 1.

Figura 1: Medidas adotadas em dedos de banana para o processo de classificação

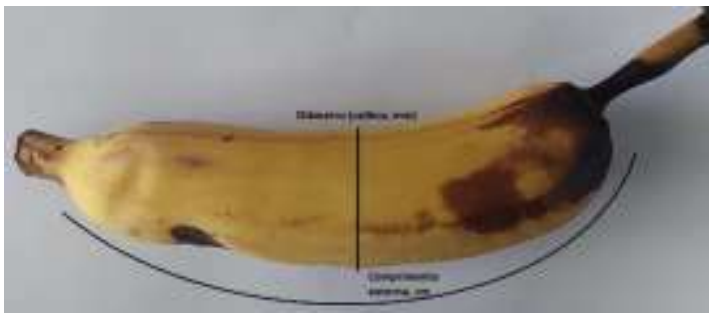

Fonte: Foto da amostra seguindo orientações de PBMH \& PIF (2006).

Com base no valor da circunferência, calculou-se o diâmetro do dedo de banana, para a classificação dos dedos entre as categorias Extra, I, II e III, Eq 1.

$\mathrm{D}=\mathrm{C} / \Pi$

Em que, $\mathrm{D}=$ diâmetro; $\mathrm{C}=$ Circunferência; $\Pi=3,1415$

Para avaliação das subclasses seguiu-se as orientações da PBMH \& PIF (2006), observando a escala de maturação, assim como para o cálculo dos defeitos foi realizado seguindo a tabela constante na cartilha de Normas de Classificação e aplicando o conceito de defeitos leves e graves.

\subsection{Critérios de classificação da banana Cavendish}

As normas de classificação são a base para a modernização da comercialização e da transparência nas relações comerciais (PBMH \& PIF, 2006; Rocha et al., 2018). A cartilha de Normas de Classificação da Banana, apresenta como um dos critérios de qualidade dos frutos a ausência de defeitos, assim as categorias descrevem a qualidade de um lote de banana, e de acordo com um calibre mínimo exigido por fruto. Dessa forma, cabe ao produtor e/ou varejista analisar os frutos ofertados e eliminar os produtos com defeitos graves, antes do seu destino para o consume humano.

De acordo com PBMH \& PIF (2006), na classificação de banana deve se considerar o grupo varietal, classe, subclasse, e a qualidade. Ainda segundo o PBMH \& PIF (2006):

1. A classe da banana é determinada pelo comprimento do fruto (em cm), sendo 6 (Maior que 6 até 9), 9 (Maior que 9 até 12), 12 (Maior que 12 até 15); 15 (Maior que 15 até 18), 18 (Maior que 18 até 22), 22 (Maior que 22 até 26) e 26 (Maior que 26);

2. A subclasse garante a homogeneidade de maturação (escala de Von Loesecke): totalmente verde (1), verde com traços amarelos (2), mais verde do que amarelo (3), mais amarelo do que verde (4), amarelo com ponta verde (5), amarelo (6) e amarelos com áreas marrons (7); 
3. As categorias correspondem a análise dos defeitos, podendo ser ausentes, leves e graves. Ainda para cada categoria, há um calibre mínimo exigido por fruto (mm).

Com relação aos defeitos considerados graves, citam-se a ponta de charuto, podridão, amassado, dano profundo, maturação precoce, fruto passado ou com traças. Os defeitos graves são "prejudiciais à saúde, nos quais inviabilizam o consumo e depreciam muito a aparência e o valor do produto", já com relação aos defeitos leves, tem-se a "ausência de dedos, desenvolvimento diferenciado, geminado e restos florais, não impedem o consumo, mesmo assim depreciam o valor do produto" (PBMH \& PIF, 2006).

\subsection{Análise de dados}

Após a coleta os dados foram tabulados em planilha eletrônica para o processamento da estatística. Assim, para as variáveis quantitativas foi realizado o estudo da normalidade aplicando o teste Shapiro-Wilk para verificar o comportamento das variáveis e se a distribuição seguia semelhante a distribuição gaussiana. Logo, para melhor compreensão dos dados aplicou-se a estatística descritiva univariada e, posterior, para comparar as classes de dedos de bananas foi processada a estatística não-paramétrica Kruskal-Wallis e o teste Dunn para comparações múltiplas a 5\% de significância.

Da mesma forma, para comparar as variáveis qualitativas foi aplicado a estatística $\chi^{2}$, com base nos valores observados e esperados, sendo considerado o nível de significância de 5\%. O processamento estatístico foi realizado em MS Excel e pelo suplemento do Excel, Real Statistics Resources.

\section{Resultados e Discussão}

Durante a coleta de dados teve um dedo de banana não localizado, resultando em valor missing no banco de dados. Assim, um total de 239 dedos de bananas da rede varejista de municípios pertencentes a região do Alto Tietê foram analisados, totalizando 37,62 Kg, média de 156,49 \pm 39,62 gramas/dedo, Tabela 1 . O preço médio do dedo de banana na região foi de 0,53 $\pm 0,21$. No estudo de Soares et al. (2020), apresentaram que a aceitação da banana no mercado brasileiro ultrapassa os valores nutricionais da fruta, inclui também as questões de preços acessíveis e praticidade no seu consumo (facilidade na higienização e remoção da casca).

Tabela 1: Resumo geral estatístico dos parâmetros de qualidade de dedos de bananas.

\begin{tabular}{ccccccc}
\hline Variáveis & Média & DV & Amplitude & Máximo & Mínimo & CV\% \\
\hline Preço & 0,53 & 0,21 & 1,20 & 1,36 & 0,16 & 39,79 \\
Peso bruto & 156,49 & 39,62 & 191 & 278 & 87 & 25,32 \\
Peso casca & 57,56 & 12,52 & 61 & 91 & 30 & 21,75 \\
Peso líquido & 98,93 & 30,11 & 161 & 214 & 53 & 30,43 \\
Comprimento & 21,11 & 2,35 & 10 & 26 & 16 & 11,13 \\
Calibre & 37,06 & 3,08 & 17,51 & 47,77 & 30,25 & 8,32 \\
\hline
\end{tabular}

Fonte: Autores (2021).

A partir da Tabela 1 é possível verificar que entre as variáveis estudadas, o preço apresentou alta variação entre os supermercados da região do Alto Tietê ( $40 \%)$, assim como o peso líquido ( $30,5 \%)$, sendo encontrados dedos de banana por 
$\mathrm{R}$ \$ 0,16 até $\mathrm{R} \$ 1,36$ e peso líquido. Entretanto, as variáveis que apresentaram baixa variação foram calibre ( 8,5\%) e comprimento $(\sim 11 \%)$.

Com base na literatura, os dedos de bananas coletados foram distribuídos por classes de 15, 18 e 22, assim, os dedos da amostra apresentaram comprimento acima de 15 e abaixo e/ou igual a $26 \mathrm{~cm}$ (PBMH \& PIF, 2006). Observou-se diferenças estatísticas e significantes $(\mathrm{p}<0,05)$ entre as classes de bananas quanto aos critérios de qualidade peso bruto, líquido, casca, comprimento e calibre. Já para o preço do dedo da banana foi observada diferença apenas entre as classes 15 e 18 e/ou 22 , mas não se obteve mesmo resultado entre as classes 18 e 22 (Tabela 2).

Tabela 2: Comparação estatística dos critérios da qualidade entre as classes de dedos de bananas.

\begin{tabular}{|c|c|c|c|}
\hline Variáveis & $\begin{array}{l}\text { Classe } 15 \\
\qquad \mathrm{n}(35)\end{array}$ & $\begin{array}{l}\text { Classe } 18 \\
\text { n(129) }\end{array}$ & $\begin{array}{c}\text { Classe } 22 \\
\text { n(75) }\end{array}$ \\
\hline Preço & $0,39 \pm 0,14^{\mathrm{a}}$ & $0,54 \pm 0,19^{b}$ & $0,60 \pm 0,25^{b}$ \\
\hline Peso bruto & $108,85 \pm 12,69^{a}$ & $146,86 \pm 25,23^{b}$ & $195,29 \pm 33,36^{\mathrm{c}}$ \\
\hline Peso casca & $44,25 \pm 8,84^{\mathrm{a}}$ & $56,14 \pm 9,96^{\mathrm{b}}$ & $67,34 \pm 10,63^{\mathrm{c}}$ \\
\hline Peso líquido & $64,57 \pm 9,17^{\mathrm{a}}$ & $90,71 \pm 20,01^{\mathrm{b}}$ & $127,94 \pm 27,40^{c}$ \\
\hline Comprimento & $17,31 \pm 0,72^{\mathrm{a}}$ & $20,60 \pm 1,13^{\mathrm{b}}$ & $23,78 \pm 0,92^{\mathrm{c}}$ \\
\hline Calibre & $33,95 \pm 1,95^{\mathrm{a}}$ & $36,83 \pm 2,90^{\mathrm{b}}$ & $38,94 \pm 2,45^{\mathrm{c}}$ \\
\hline
\end{tabular}

a,b,c letras semelhantes não são estatisticamente significantes pelo teste Dunn $(\mathrm{p}<0,05)$. Fonte: Autores (2021).

Com relação a classificação por calibre mínimo, 95\% dos dedos apresentaram calibre igual ou superior a $32 \mathrm{~mm}$ (categoria Extra), contra 5\% que foram classificados na categoria I (calibre entre 30 e 31,99 mm).

\subsection{Avaliação dos defeitos nos dedos de banana Cavendish}

Com relação à análise das subclasses dos dedos de bananas, observou-se que a maioria foram classificados nas subclasses 7 (cerca de 37,5\%) e 6 (35\%), Figura 2. De acordo com a escala de maturação de Von Loesecke, os dedos classificados nas subclasses 6 e 7 se referem aqueles frutos com casca totalmente amarela e amarelo com áreas marrons, respectivamente (PBMH \& PIF, 2006). Estes resultados foram corroborados por Rocha et al. (2018), que obtiveram em seus estudos que as bananas de retorno foram enquadradas nas subclasses 7 e as bananas do mercado na subclasse 6 . Ainda de acordo com os autores um fator de qualidade da banana é a sua coloração. Uma das vantagens da banana é sua versatilidade quanto ao uso na culinária doméstica como na fabricação de novos produtos (Soares et al., 2020), podendo ser utilizada para produção de diversos alimentos para o consumo humano. 
Figura 2: Distribuição dos dedos de banana por subclasses

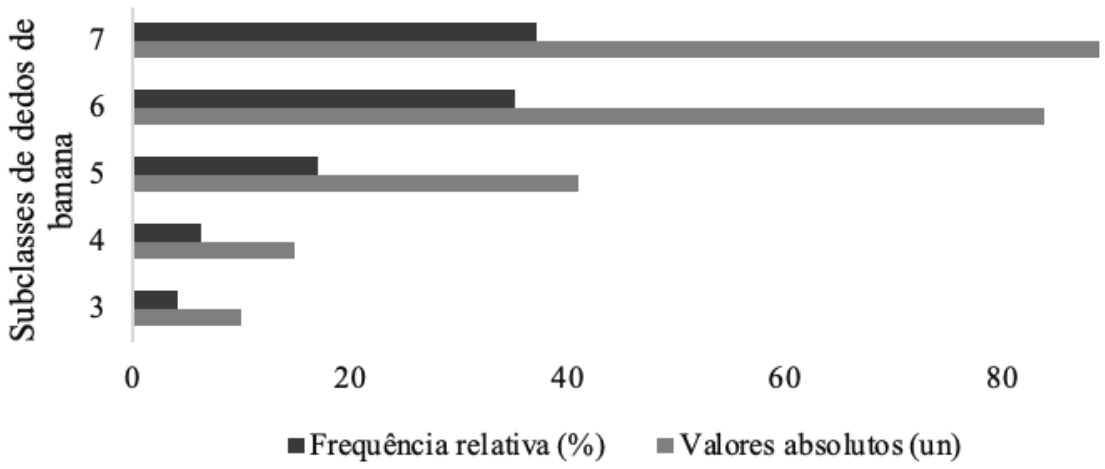

100

-Frequência relativa (\%) $\quad$ Valores absolutos (un)

Fonte: Autores (2021)

Em termos gerais, do total de dedos de banana analisados, constatou-se que $62 \%$ apresentaram defeitos de grau II e III contra $38 \%$ que não apresentou defeitos e/ou defeitos leves. Foi observado diferença estatística e significante para a análise dos defeitos de dedos de banana por município.

Comparando as amostras obtidas entre os municípios, notou-se que Itaquaquecetuba apresentou a menor taxa de defeitos entre os dedos de bananas, sendo a maioria classificado na categoria Extra e nenhum apresentou defeitos graves. Enquanto Biritiba Mirim teve cerca de 79,5\% de seus frutos classificados na categoria III, considerados defeitos graves, com os dedos passados e sinais de podridão, Tabela 3.

Tabela 3: Avaliação dos defeitos detectados em dedos de banana, por município

\begin{tabular}{|c|c|c|c|c|}
\hline \multirow[t]{2}{*}{ Municípios } & \multicolumn{4}{|c|}{ Categorias } \\
\hline & Extra & I & II & III \\
\hline Biritiba Mirim & 15,38 & - & 5,13 & 79,49 \\
\hline Guarulhos & 45 & - & - & 55 \\
\hline Itaquaquecetuba & 65 & 2,5 & 32,5 & - \\
\hline Mogi & 37,5 & 2,5 & - & 60 \\
\hline Poá & 37,5 & - & 45 & 17,5 \\
\hline Suzano & 27,5 & - & 22,5 & 50 \\
\hline
\end{tabular}

$\chi^{2}=98,11 ;$ p-valor < 0,05. Fonte: Autores (2021).

Como já citado, a banana está entre as frutas mais consumidas pelos brasileiros e a não fiscalização e/ou cuidados por parte dos varejistas na oferta de frutos com defeitos graves, pode causar problemas sanitários e de saúde. Além disso, os frutos não vendidos se tornam quebra e gera desperdício para o varejo, ou seja, toda a cadeia perde com a falta de monitoramento dos frutos. Dessa forma, uma alternativa para redução das perdas no setor de hortifruti é o monitoramento dos frutos expostos para venda, com a retirada diária de amostras para sua classificação, auxiliando na tomada de decisão sobre manter os frutos expostos para venda ou direcioná-los para a produção de alimentos processados. Soares et al. (2020), apontaram que 74\% dos 
entrevistaram consomem produtos derivados da banana desde iogurte e sorvetes a massas e bolos.

Adotar ferramentas da qualidade e aplicá-las adequadamente requer tempo e investimento em desenvolvimento de procedimento e treinamento de mão de obra empregada. Assim, empresas do ramo alimentício adotam os procedimentos de qualidade de programas obrigatórios, relacionados a vigilância sanitária e inspeção estadual, mas não praticam os programas de gestão da qualidade (Mannes et al., 2018).

A análise de defeitos por lotes, verificou que do total de 12 apenas 02 lotes foram considerados dentro do padrão de qualidade para comercialização, conforme as Normas de Classificação da Banana, do Programa Brasileiro para Modernização da Horticultura \& Produção Integrada de Frutas (PBMH \& PIF, 2006). Soares et al. (2020), alertaram que os consumidores observam muito a aparência do fruto, assim, os produtores e os envolvidos na distribuição de banana devem buscar por alternativas para não causar danos mecânicos, mantendo os frutos com uma aparência mais agradável e transporte de melhor qualidade. De acordo com Hsiao et al. (2017) e Macheka et al. (2017), atenção especial deve-se aplicar ao gerenciamento da qualidade e segurança alimentar de alimentos frescos que requerem cuidados das particularidades de perecibilidade e sensibilidade a temperatura natural.

Estudos revelam que a falta de planejamento e gerenciamento das operações de transporte afetam a qualidade dos alimentos, principalmente, dos frescos que requerem cuidados especiais, como aplicação da cadeia logística do frio (Trienekens \& Wognum, 2013; Hsiao et al., 2017; Macheka et al., 2017). A banana é um produto de entrada direta nos varejos e dos 239 dedos avaliados, não houve nenhum rótulo ou etiqueta informando as origens dos produtos, apenas o código de barras disponibilizado na pesagem do fruto para controle do próprio varejista, mas nada relacionado a qualidade e rastreio, o que contraria questões de transparência do varejista para com o consumidor. Os estudos de Kondo (2010), tratam da importância do controle da qualidade e da aplicação do sistema de rastreabilidade para a cadeia de hortifruti, gerando credibilidade para cadeia produtiva. Ainda, a falta de controle não permite a seleção de produtos para diferentes usos, em função da qualidade, com diferenciação de preços e redução de despesas na comercialização.

Considerando o preço médio por categoria, nota-se que independente dos dedos de bananas apresentarem defeitos graves e/ou leves, variou entre de $\mathrm{R} \$ 3,29$ a $\mathrm{R} \$ 3,74$. Este resultado indica que o varejista não aplica redução de preço devido a qualidade das frutas. Entretanto, no dia da coleta, observou-se ofertas de bananas pelo valor de $\mathrm{R} \$ 4,18$ o quilo e qualidades impróprias ao consumo (frutos com defeitos graves), e também ofertas no valor de R \$ 1,99 com frutos adequados para demanda final de um supermercado.

Os estudos de Soares et al. (2020), reportam que os fatores que mais influenciam os consumidores na hora da compra são a textura e aparência externa da fruta, e complementa que há uma valorização do tamanho médio e o grande. Dessa forma, fica evidenciado que os defeitos encontrados nos dedos de banana são fatores que prejudicam a comercialização e a escolha da fruta na hora da compra, resultando em desperdícios para a cadeia. Ainda foi possível notar que os supermercados mais próximos aos centros de distribuição e entreposto oferecem bananas de melhor qualidade, seguindo padrões de tamanhos e maturação. Enquanto os supermercados mais distantes da capital oferecem frutas em tamanhos e maturação variáveis e, muitas das vezes, em condições inapropriadas ao consumo humano.

\subsection{Proposta para redução das perdas na cadeia de hortaliças}

Atualmente, a prática de mercado tem utilizado o processo de classificação manual para separar os produtos com padrões de comercialização daqueles que não apresentam tais características. Isto tem levado muitos produtos a serem rejeitados para a comercialização e não são enviados para o mercado. Dessa forma, o processo de classificação quando desenvolvido como suporte à tomada de decisão da cadeia e com proposito de destinação correta da produção, contribuiu para a redução dos desperdícios, além de apresentar diversos benefícios como apontados por Kondo (2010) e serve para a 
identificação de produtos inapropriados ao consumo (Dabbene \& Gay, 2011), Figura 3.

Observando a Figura 1, percebe-se que os produtos classificados como "Classe 1" são aqueles que apresentam os padrões de qualidade aceitos pelo mercado, logo devem ser destinados para comercialização. Enquanto os produtos da "Classe 2", são os produtos que não apresentam os padrões de qualidade e perderam valor de mercado, mas estão bons e adequados para o consumo humano, assim, devem ser enviados para a indústria de alimentos, para seu processamento. Rocha et al. (2018), corroboram com esta afirmação dado que expõem a industrialização como uma opção para aproveitamento dos produtos com aparência comprometida ao consumo na forma in natura.

Os produtos que estão fora dos padrões de qualidade e/ou não estão adequados para o consumo humano (Classe 3), podem ser destinados a alimentação animal e devem ser enviados para a indústria de ração; ainda, os produtos que não estão adequados nem para consumo humano ou animal, devido apresentar grau de putrefação, devem ser enviados para a produção de adubo orgânico.

Dessa forma, um processo de classificação integrado ao fluxo logístico de distribuição se torna crucial para atender as necessidades especiais de rapidez de produtos perecíveis e contribuir com a redução das perdas da cadeia (Hsiao et al., 2017). Yap et al. (2017), reportam o aproveitamento de bananas rejeitadas para comercialização, devido não apresentarem o tamanho requerido pelo controle de qualidade para a produção de purê na Austrália.

Figura 3: Proposta de destinação da produção baseada no processo de classificação

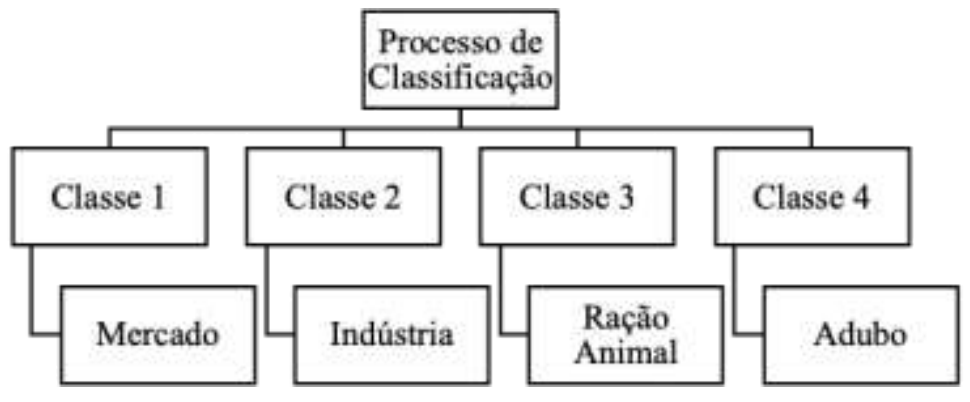

Fonte: Autores (2021).

A não realização de uma classificação das bananas interfere diretamente na qualidade e rastreio de toda cadeia, ocorrendo o mesmo com os demais produtos de hortifrúti no Alto Tiete. Com isso, não se atende as necessidades de produtos perecíveis, além de complementar o dito em Machecka et al. (2017) sobre os principais desafios da logística aplicados as cadeias de hortifrúti, visto que os produtos encontrados apresentaram fatores que certamente contribuem com aumento das perdas da cadeia, atenuando o desperdício.

Mediante ao apresentado, nota-se que os resultados obtidos nesta pesquisa são muito distantes do esperado e exibido na Figura 4, onde os frutos encontrados pelo consumidor final não passam por um processo de classificação. Seguindo a proposta de destinação da produção baseada no processo de classificação, conclui-se que dos 239 dedos de bananas, 148 unidades $(61,92 \%)$ tiveram uma destinação incorreta, sendo que ao invés de disponíveis em mercados, deveriam ter sido destinados a indústria, ração animal ou adubo. 
Figura 4: Processo de classificação e destinação correta dos dedos de bananas.

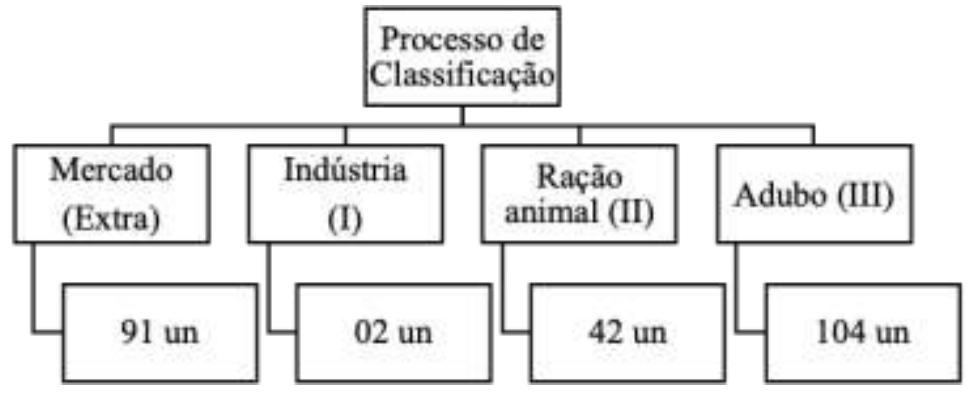

Fonte: Autores (2021).

De acordo com Soares et al. (2020), a banana pode ser matéria-prima para a produção de diversos pratos, tais como: iogurte, doces, massas, bolos, sorvete, farinha, passas, flocos, barras de cereais, desidratada, chips, purê, tortas, etc. Isso só reforça que o processo de classificação auxilia na destinação correta dos alimentos para evitar desperdícios, assim, a análise previa dos frutos e o tratamento adequado possibilitaria a produção de mais alimentos saudáveis e a garantia da segurança alimentar.

\section{Conclusão}

O processo de classificação de banana permeia a cadeia produtiva e requer um novo comportamento de todos os envolvidos. Contudo, pelos resultados da pesquisa ficou evidenciado que não basta realizar a classificação apenas na transferência das frutas entre fornecedor e comprador, é necessário manter o monitoramento dos alimentos durante a exposição nas bancas do varejo/atacado, garantindo a segurança alimentar, redução de desperdício de alimentos com a destinação correta de frutas para a produção de alimentos processados.

Avaliando os critérios de classificação de banana Cavendish, por meio de medições do comprimento, calibre, pesos e as condições do fruto para o consumidor final, tem-se como principal resultado que o varejo na região do Alto Tietê oferta bananas de tamanhos médio e grandes (classes 15, 18 e 22), a maioria com grau de amadurecimento entre as subclasses 6 e 7 e categorizados como Extra (calibre superior a $32 \mathrm{~mm}$ ). Contudo, constatou-se também a presença de muitos defeitos por lote o que afeta a qualidade do varejo da região.

Como estratégia alternativa, propõem o monitoramento do tempo de prateleira da fruta e a realização do processo de classificação diário, selecionando amostras e verificando as condições dos frutos, se permanecem expostos a venda ao consumidor final ou se devem seguir para produção de alimentos, ração ou compostagem. No estudo, foi observado que cerca de $62 \%$ dos dedos de bananas não deveriam permanecer no varejo para sua comercialização.

Este artigo apresentou a importância do monitoramento da qualidade das frutas ofertadas no varejo para consumo, por meio dos métodos de classificação e da análise das características externas de bananas, contudo, recomenda-se como estudo futuro uma análise conjunta das características externas e internas, para verificar se os danos externos impactam nas perdas de qualidade nutricional da banana.

\section{Agradecimentos}

Os autores agradecem a bolsa recebida pelo PIBIFSP-IFSP, Câmpus Suzano. 


\section{Referências}

Almeida, G. V. B. (2021). A importância da pós-colheita: hortifrutis são alimentos vivos e precisam de cuidados especiais. Consultado a 21 de 10 de 2021. https://ceagesp.gov.br/entrepostos/servicos/artigos-estudos-e-publicacoes/artigos/a-importancia-da-pos-colheita/.

Camargo Filho, W.P. \& Camargo, F.P. (2019). PIB da produção de hortaliças no Estado de São Paulo, 2017. Revista de Agronegócio, Instituto de Economia Agrícola. Consultado a 11 de 10 de 2021. https://revistadeagronegocios.com.br/iea-instituto-de-economia-agricola-pib-da-producao-de-hortalica\&\#8230

CEAGESP, Companhia de Entrepostos e Armazéns Gerais de São Paulo. (2021). Carta anual de políticas públicas e governança corporativa 2020. Consultado a 11 de 10 de 2021. http://ceagesp.gov.br/acesso-a-informacao/governanca/carta-anual-de-politicas-publicas-e-governanca-corporativa/

CNA, Confederação da Agricultura e Pecuária do Brasil. (2020). Pib do agronegócio alcança participação de 26,6\% no Pib brasileiro em 2020. Consultado a 11 de 10 de 2021. https://www.cnabrasil.org.br/assets/arquivos/boletins/sut.pib_dez_2020.9mar2021.pdf

Dabbene, F. \& Gay, P. (2011). Food traceability systems: Performance evaluation and optimization. Computers and Electronics in Agriculture, 75 (1), 139146. https://doi.org/10.1016/j.compag.2010.10.009

Franco, C.; Pedersen, S. M.; Papaharalampos, H. \& Ørum, J. E. (2017). The value of precision for image-based decision support in weed management. Precision Agriculture, 18 (3), 366-382. https://doi.org/10.1007/s11119-017-9520-y

Hsiao, Y.-H.; Chen, M.-C. \& Chin, C.-L. (2017). Distribution planning for perishable foods in cold chains with quality concerns: Formulation and solution procedure. Trends in Food Science \& Technology, 61, 80-93. https://doi.org/10.1016/j.tifs.2016.11.016

IBGE, Instituto Brasileiro de Geografia e Estatística. (2018). População estimanda: Arujá, Biritiba Mirim, Ferraz de Vasconcelos, Guararema, Guarulhos, Itaquaquecetuba, Mogi das Cruzes, Poá, Salesópolis, Santa Branca, Santa Isabel e Suzano. https://cidades.ibge.gov.br/

IBGE, Instituto Brasileiro de Geografia e Estatística. (2020). Área, Produção e Rendimento Médio - Confronto das safras de 2020 e das estimativas para 2021. https://www.ibge.gov.br/estatisticas/economicas/agricultura-e-pecuaria/9201-levantamento-sistematico-da-producao-agricola.html?=\&t=resultados

Kondo, N. (2010). Automation on fruit and vegetable grading system and food traceability. Trends in Food Science \& Technology, 21 (3), 145-152. https://doi.org/10.1016/j.tifs.2009.09.002

Lindblom, J.; Lundström, C.; Ljung, M. \& Jonsson, A. (2017). Promoting sustainable intensification in precision agriculture: review of decision support systems development and strategies. Precision Agriculture, 18 (3), 309-331. https://doi.org/10.1007/s11119-016-9491-4

Macheka, L.; Spelt, E.; Vorst, J. G.A.J. van der. \& Luning, P. A. (2017). Exploration of logistics and quality control activities in view of context characteristics and postharvest losses in fresh produce chains: A case study for tomatoes. Food Control, 77, 221-234. https://doi.org/10.1016/j.foodcont.2017.02.037

Mannes, J. F.; Pitz, A.; Fraga, I. S. \& Martins, Z. B. (2018). Gestão da qualidade no ramo alimentício: um estudo de caso em um frigorifico. Research, Society and Development, 7(3), 1-21, e1273285.

PBMH \& PIF, Programa Brasileiro para a Modernização da Horticultura \& Produção Integrada de Frutas. (2006). Normas de Classificação de Banana [Banana Classification Standards]. CEAGESP, Documentos, 29.

Perovano, D. G. (2016). Manual de metodologia da pesquisa científica. Intersaberes. ISBN 978-85-5972-021-1

Rocha, C. S.; Silva, D. M. C. T.; Simonato, A. L. \& Gorayeb, R. C. C. (2018). Classificação de banana nanica (Musa Cavendishi) para a produção de bananas passas [Classification of dwarf banana (Musa Cavendishi) for the production of raisin bananas]. Mobilizar o Conhecimento para Alimentar o Brazil, 529-533.

Soares, J. M. S.; Silva, M. S.; Nascimento, F. S.; Gonçalves, Z. S.; Souza, T. R.; Rodrigues, T. C.; Sousa, Y. M. \& Souza, H. B. F. (2020). Preferências dos consumidores de banana quanto à qualidade do fruto em Cruz das Almas - BA. Revista Agrária Acadêmica, 3 (6), 86-95. https://doi.org/10.32406/v3n6/2020/86-95/agrariacad

Trienekens, J. \& Wognum, N. (2013). Requirements of supply chain management in differentiating European pork chains. Meat Science, 95 (3), 719-726. https://doi.org/10.1016/j.meatsci.2013.03.035

Yap, M.; Fernando, W.; Brennan, C.; Jayasena, V. \& Coorey, R. (2017). The effects of banana ripeness on quality indices for puree production. $\{L W T\}$ - Food Science and Technology, 80, 10-18. https://doi.org/10.1016/j.1wt.2017.01.073 